\title{
Cadaveric Study of Anatomic Far Distal Musculocutaneous and Median Nerve Communication
}

\author{
Namath S. Hussain ${ }^{1}$ \\ 1. Department of Neurosurgery, Loma Linda University Medical Center \\ $\square$ Corresponding author: Namath S. Hussain, namath.hussain@gmail.com \\ Disclosures can be found in Additional Information at the end of the article
}

\section{Abstract}

One of the more commonly reported variations in musculocutaneous and median nerves is a communication between the two. Such communications have been noted in the literature for well over a century, and numerous studies have attempted to quantify their rate of incidence and classify their different patterns. Most communications occur close to the brachial plexus in the proximal arm; communications distal to the musculocutaneous nerve perforation of the coracobrachialis have been reported with less frequency, and several rare and unique communication patterns have been reported as case studies. This paper describes a case of a communicating branch between the musculocutaneous and median nerves, distal to the origin of the brachialis muscle. This communication was found during a routine educational dissection and is closer to the level of the elbow than previously documented communications that are distal to the musculocutaneous perforation of the coracobrachialis. Identification and documentation of the variety of musculocutaneous and median nerve communications is relevant to clinical diagnosis of peripheral nerve pathologies, as well as for planning surgical approaches and procedures.

Categories: Neurosurgery, Orthopedics

Keywords: median nerve, musculocutaneous nerve

\section{Introduction}

Variations in anatomic patterns of both the musculocutaneous (MC) and median nerves have been described in the literature for well over a century [1]. One of the most commonly reported variations is a communication between the two nerves as depicted in Figure 1. 


\section{Cureus}

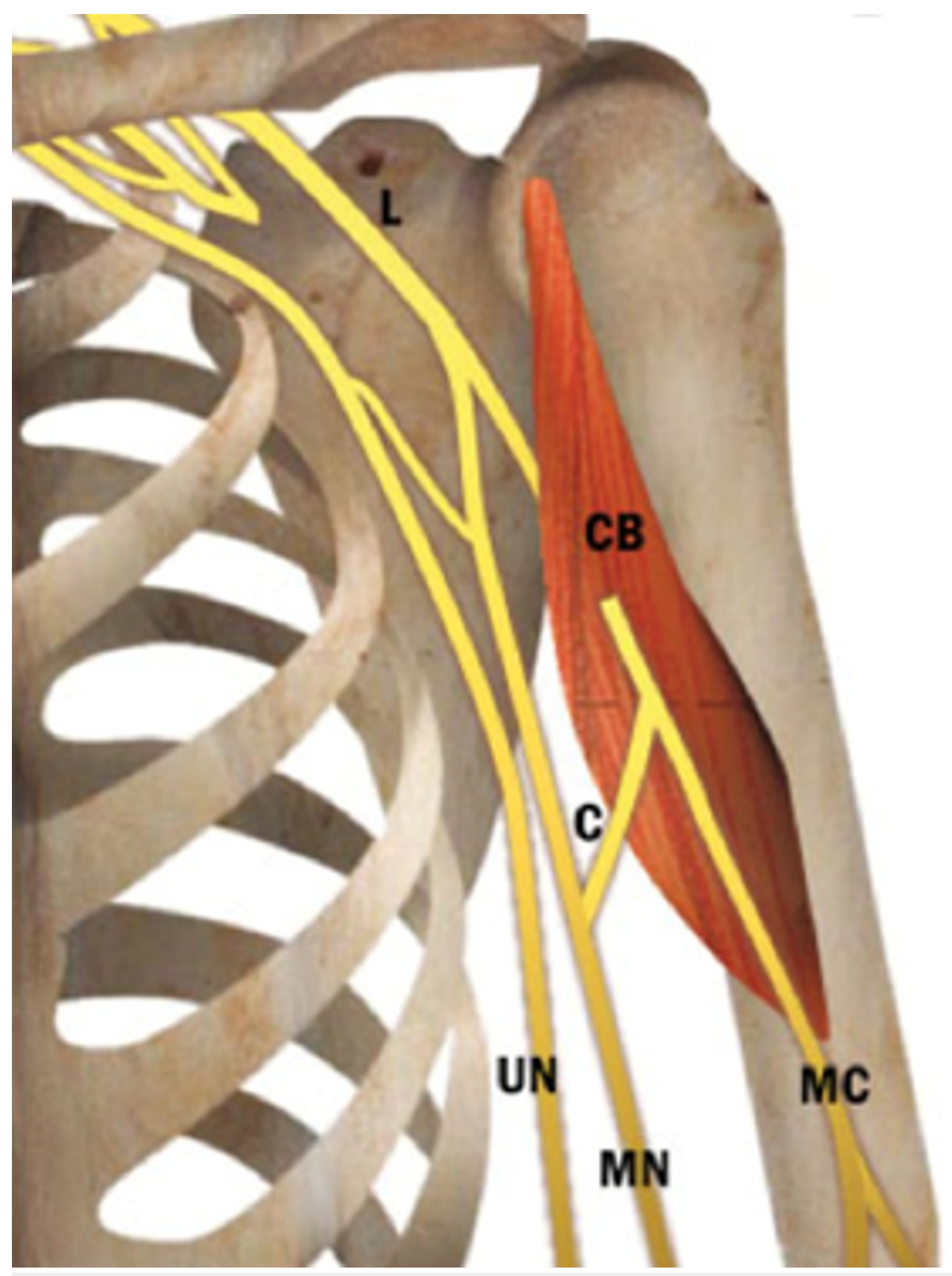

FIGURE 1: Communication Between MC and Median Nerves

Such variations have been found to occur in $10 \%-43 \%$ of cadaver upper limbs [15]. Classification of these variations has been the focus of numerous studies. Proposed schemes have included parameters that describe different variations including the number of communications, direction of communication (MC to median or vice-versa), location of communication relative to the MC perforation of the coracobrachialis (CB), and the presence of other contributing brachial plexus or musculoskeletal abnormalities that may be contributing factors [1-2, 5-6]. Awareness and effective classification of MC nerve variations is of clinical importance both in terms of diagnosis of peripheral nerve pathology and for surgical interventions and approaches due to possible increased risk of nerve injury. 


\section{Cureus}

\section{Case Presentation}

A unilateral communication between the MC and median nerves was observed during a cadaveric study of the brachial plexus, examining anatomic variants. The brachial plexus and associated peripheral nerves were carefully dissected and identified in order to assess their anatomical relationships. The variation was recorded and documented by neurosurgeons.

The communication was found in the left arm, branching off from the $\mathrm{MC}$ after piercing the CB muscle, distal to both the insertion of the $\mathrm{CB}$ and the origin of the brachialis muscle. The branch continued medially and distally to join the median nerve as shown in Figure 2.

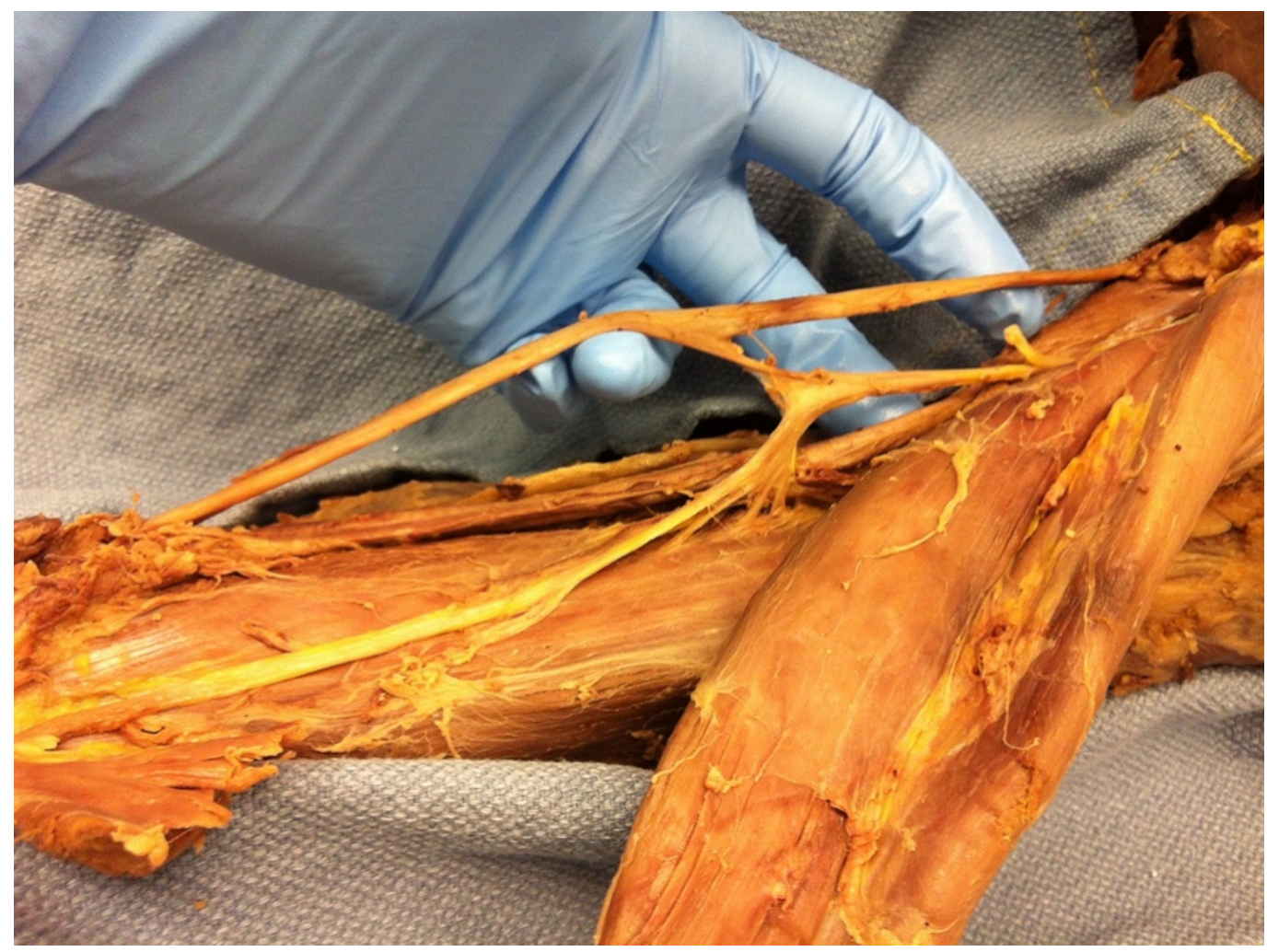

FIGURE 2: Cadaveric Demonstration of Far Distal Communication Between MC and Median Nerves

Top of figure is lateral, bottom of figure is medial, right side of figure is proximal, left side of figure is distal.

The communication was six inches from the coracoid and four inches from the elbow flexion crease. No other abnormalities of the brachial plexus, median nerve, $\mathrm{MC}$ nerve, or other peripheral nerves were found and no communication was present in the right arm.

\section{Discussion}

Communications between the $\mathrm{MC}$ and median nerves are more often found proximal to the MC perforation of the $\mathrm{CB}$, closer to the brachial plexus [2]. Communications distal to the $\mathrm{MC}$ perforation of the $\mathrm{CB}$ are classified as $1 \mathrm{~A}$ to $1 \mathrm{D}$ variation by a system proposed by GuerriGuttenberg and Ingolotti [2]. Such communications have been found more proximally, and previous reports of this type of communication have not described a communication distal to 
the CB insertion or distal to the origin of the brachialis muscle $[1,3]$.

Communicating branches between the MC and median nerves are thought to be a result of fibers that normally run through the lateral root of the median nerve and instead join it distally via the MC nerve. This phenomenon is described here as more distally located than typically reported and should be considered in relevant clinical settings such as biceps tendon repairs, given the high incidence (up to $43 \%$ ) of similar communications.

This classification indicates a more rare variation, as the majority of communications have been found to occur proximal to the $\mathrm{CB}$ and at the level of the brachial plexus [7-8]. Communications from the MC nerve to the median have been reported as being distal to the MC perforation of the $\mathrm{CB}$ in $7.7 \%-56 \%$ of all cases, indicating a lack of consensus on its prevalence [1-2].

The Guerri-Guttenberg and Ingolotti classification system does leave some gray area within this $1 \mathrm{~A}$ to $1 \mathrm{D}$ category of variation. Communications in this classification have a proximal boundary-the perforation of the $\mathrm{CB}$-but no distal boundary, making it difficult to categorize the location of the communication. Other classification systems for MC variations have generally been less robust and also do not specify the level of a communicating branch that is distal to the $\mathrm{CB}$.

Although several studies have described a communication distal to the MC perforation of the $\mathrm{CB}$, none have described a communication distal to the $\mathrm{CB}$ insertion or distal to the origin of the brachialis muscle. Representations of a communication distal to the $\mathrm{MC}$ perforation of the CB have typically been shown to be proximal to the insertion of the CB $[1-2,5]$. There have been rare case reports of communications at or distal to the cubital fossa including hooking around the median artery in the forearm [9].

Traditionally with an upper plexus injury, elbow flexion is restored with an Oberlin transfer, which involves transfer from the ulnar nerve to the biceps motor nerve. MacKinnon felt that this transfer was not robust enough to provide functional improvement and suggested transferring median nerve branches to the brachialis motor branch of the MC nerve in addition to the Oberlin transfer [10]. Because that transfer occurs in the mid to distal arm and involves the median and MC nerves, knowledge of this variant is important to surgeons doing this procedure.

Communicating branches between the MC and median nerves are thought to be related to embryologic development and a result of fibers that normally run through the lateral root of the median nerve and instead join it distally via the MC nerve [2].

\section{Conclusions}

This is the first report of a specific anatomical variant of a far distal MC and median nerve communication. This variant was documented in a controlled anatomic cadaveric study. This finding has implications for surgeons dealing with the local anatomy, including orthopedic surgeons performing biceps tendon repairs and neurosurgeons performing Oberlin nerve transfers.

\section{Additional Information \\ Disclosures}

Human subjects: Consent was obtained by all participants in this study. Conflicts of interest: The authors have declared that no conflicts of interest exist. 


\section{References}

1. Choi D, Rodriguez-Niedenfuhr M, Vazquez T, Parkin I, Sanudo JR: Patterns of connections between the musculocutaneous and median nerves in the axilla and arm. Clin Anat. 2002, 15:11-17. 10.1002/ca.1085

2. Guerri-Guttenberg RA, Ingolotti M: Classifying musculcutaneous nerve variations . Clin Anat. 2009, 22:671-683. 10.1002/ca.20828

3. Maeda S, Kawai K, Koizumi M, Ide J, Tokiyoshi A, Mizuta H, Kodama K: Morphological study of the communication between the musculocutaneous and median nerves. Anat Sci Int. 2009, 84:34-40. 10.1007/s12565-008-0005-y

4. Uysal II, Karabulut AK, Buyukmumcu M, Dogan NU, Salbacak A: The course and variations of the branches of the musculocutaneous nerve in human fetuses. Clin Anat. 2009, 22:337-345.

10.1002/ca.20734

5. Venieratos D, Anagnostopoulou S: Classification of communications between the musculocutaneous and median nerves. Clin Anat. 1998, 11:327-331. 10.1002/(SICI)10982353(1998)11:5<327::AID-CA6>3.0.CO;2-M

6. Loukas M, Aqueelah H: Musculocutaneous and median nerve connections within, proximal and distal to the coracobrachialis muscle. Folia Morphol (Warsz). 2005, 64:101-108.

7. Johnson EO, Vekris M, Demesticha T, Soucacos PN: Neuroanatomy of the brachial plexus: normal and variant anatomy of its formation. Surg Radiol Anat. 2010, 32:291-297.

10.1007/s00276-010-0646-0

8. Chitra R: Various types of intercommunications between musculocutaneous and median nerves: an analytical study. Ann Indian Acad Neurol. 2007, 10:100-104. 10.4103/09722327.33217

9. Agarwal S, Tuli A, Raheja S: Communication between median and musculocutaneous nerves in the forearm hooking around the origin of the median artery: a rare variation. Anat Sci Int. 2011, 86:175-177. 10.1007/s12565-011-0105-y

10. Ray WZ, Pet MA, Yee A, Mackinnon SE: Double fascicular nerve transfer to the biceps and brachialis muscles after brachial plexus injury: clinical outcomes in a series of 29 cases. J Neurosurg. 2011, 114:1520-1528. 10.3171/2011.1.JNS10810 